\title{
PREPARATION OF CHIRAL ORGANIC-INORGANIC SOLID HYBRIDS. USE AS SUPPORT OF CATALYSTS IN THE ENANTIOSELECTIVE HYDROGENATION OF ETHYL PYRUVATE
}

\author{
PATRICIO REYES ${ }^{I^{*}}$, CRISTIÁN CAMPOS ${ }^{l}$ AND JOSÉ LUIS GARCÍA FIERRO ${ }^{2}$
}

\author{
${ }^{1}$ Universidad de Concepción, Facultad de Ciencias Químicas, Departamento de Fisicoquímica. Edmundo Larenas 129, 160-C, Concepción, Chile. \\ ${ }^{2}$ Instituto de Catálisis y Petroleoquímica, CSIC, Cantoblanco, 280440 Madrid, Spain
}

(Received 13 $3^{\text {th }}$ March $2007-$ Accepted $13^{\text {th }}$ June 2007)

\begin{abstract}
Chiral organic-inorganic hybrids have been prepared from 1,3,5,7-tetramethylcyclotetrasiloxane (TMCTS), divynilbenzene (DVB) and a natural alkaloid (-)-Cinchonidine (CD). Different CD quantities were inserted into the solid by hydrosilylation reaction using PtO $_{2}$ as catalyst. The obtained solids were used as supports of Ru which was deposited on the carrier from colloidal dispersion of the metal. Catalysts and supports were characterised by using Fourier Transformed Infrared Spectroscopy, X- Ray Diffraction, Electron Transmission Microscopy, $\mathrm{N}_{2}$ adsorption-desorption isotherms and X- Ray photoelectron spectroscopy. The catalysts were essayed in the hydrogenation of ethyl pyruvate at $298 \mathrm{~K}$ and 40 bar of hydrogen in a Parr-type reactor. The obtained results indicate that the catalytic activity of the hybrid catalysts is rather low and in those catalysts having CD in their structure, is possible to induce chirality towards the enantiomer R-lactate.
\end{abstract}

Keywords: Ru; Ethyl pyruvate; Cinchonidine; Hybrid catalysts; Enantioselectivity

\section{INTRODUCTION}

In the last few years an increasing interest in the application of single enantiomer of chiral compounds has been detected. This occurs specially for substances useful in the field of pharmaceuticals, agrochemicals, flavors and fragrances [1]. There are several procedures to induce the preferential production of one enantiomer, being the use of homogeneous catalysis using metal complexes with chiral ligands the most widely used. In order to overcome the problems related to the separation of catalyst from the reactant mixture (substrate, products and solvent), which limit their application at industrial scale, the immobilization of homogeneous catalysts has appeared especially attractive. However, in many cases the selectivity obtained in these heterogenised catalysts is much lower compared to the homogeneous counterpart.

Heterogeneous catalysis using metal-supported catalysts has also been used in asymmetric hydrogenations. For this purpose, the addition of a chiral molecule as modifier is necessary in order to induce the required enantioselectivity. Cinchonidine, a natural alkaloid, has been often used as modifier. This alkaloid fulfils the minimum structural requirements to induce chirality: a quinoline or naphthalene ring having a basic nitrogen in the quinuclidinic group and an $\mathrm{OH}$ group with optical activity (either with $\mathrm{R}$ or $\mathrm{S}$ configuration), being the $\mathrm{OH}$ configuration of the inducer responsible of the production of a given enantiomer [2]. The anchorage of $\mathrm{CD}$ to an inorganic support such as silica has been used as an alternative to prepare a chiral support and thus avoids the problems related to reproducibility when this kind of catalysts are used in cycling experiments [3]. A new an attractive way to get chiral catalysts is the preparation of hybrid chiral organic-inorganic solids as supports of metallic catalysts which is described in the present work and used as catalysts in the hydrogenation of ethyl pyruvate. Only few studies in this area have been reported. Pettersson et al. [4] have reported modifications of the cinchonidine alkaloid by acetylation of the $\mathrm{OH}$ group with acetic anhydride. Lämmerhofer et al. [5] have synthesised quinine and quinidine derivatives by formation of carbamates with the corresponding $\mathrm{OH}$. Mäki-Arvela et al. [6] have prepared the 11-(triethoxysilyl)-10,11-dihydrocinchonidine, with triethoxysilane and analogous silane derivatives [7] by hydrosilylation reaction [8], protecting the $\mathrm{OH}$ group of the $\mathrm{CD}$ due to its potential participation in the hydrosilylation reaction.

Figure 1 displays the structure of the compounds used in the preparation of the hybrids. As can be seen, the tetramethylcyclotetrasiloxane (TMCTS) possesses four Si-H bonds which may be used for coordination. Pinho et al. [9] have prepared hybrid solids of TMCTS with p-divinylbenzene leading to thermally stable solids of TMCTS-DVB with different DVB/TMCTS ratios.

As active metal for asymmetric hydrogenation noble metals have been widely used, being platinum the most commonly chosen. The use of Ru in heterogeneous catalysis has not been so often used in spite of their important application in homogeneous catalysis due to their special ability to generate active organometallic complexes [10]. In the traditional procedure to get metal supported catalyst, the metal component is added to the support by the traditional impregnation method followed by calcination and reduction treatments. This procedure can not be used when the support contains organic species due to the oxidation and reduction steps causes modification of the support with decomposition of the organic substances.

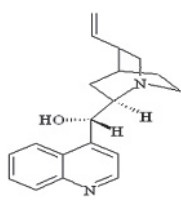

(a)

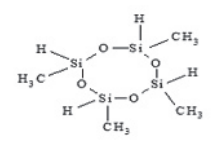

(b)

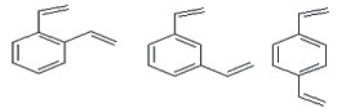

(c)
Figure 1. Structure of: (a) Cinchonidine; (b) 1, 3, 5, 7 trimethylchlorosilane and (c) divinylbenzene.

Kurihara et al. [11] have reported a general and reproducible procedure to synthesise metal colloids. Wang et al. [12] have prepared colloids of Pt, Rh y Ru using a similar procedure but modified by the addition of sodium hydroxide as a stabilizing agent.

In the present work, we have prepared and characterised chiral hybrid TMCTS-DVB-CD supports in which the TMCTS/DVB was kept constant and the amount of $\mathrm{CD}$ bond to the solid was varied. Thus, $\mathrm{CD} / \mathrm{TMCTS}$ molar ratio of $0,1 / 100,1 / 50$ and 1/10 were used. Catalysts containing $3 \mathrm{wt} \%$ of Ru on the prepared support were prepared by impregnation of the support with a colloidal dispersion of $\mathrm{Ru}$. The catalysts were characterised and used in the hydrogenation of ethyl pyruvate under similar conditions to those reported by Orito et al. [13]

\section{EXPERIMENTAL}

\section{General}

All the reactions with air sensitive reagents were carried out under inert atmosphere. THF was dried and distilled under argon from sodium benzophenone prior to use. CD ( $96 \%$ Aldrich), trimethylchlorosilane $(98 \%$ Merck), TMCTS ( $99 \%$ Aldrich) were used as received. Triethylamine and DVB were distilled under reduced pressure prior their use. The $\mathrm{PtO}_{2}$ was obtained by flowing $\mathrm{O}_{2}$ at $550 \mathrm{~K}$ on $\mathrm{H}_{2} \mathrm{PtCl}_{6}$ (Aldrich $96 \%$ ) for $3 \mathrm{~h}$. $\mathrm{RuCl}_{3} \cdot \mathrm{nH}_{2} \mathrm{O}$, Ethylene Glycol (AR grade) were supplied by Merck and used as received.

\section{Protection of $C D$}

To an ice-cooled solution of CD (1.3 g, $4.4 \mathrm{mmol})$ in THF containing triethylamine $(0.377 \mathrm{~mL}, 5.1 \mathrm{mmol})$ was added dropwise trimethylchlorosilane $(0.477 \mathrm{~mL}, 5.1 \mathrm{mmole})$ [7]. The reaction mixture was stirred for $20 \mathrm{~h}$ at room temperature and then for $2 \mathrm{~h}$ at $60^{\circ} \mathrm{C}$. The product was extracted with chloroform $(50 \mathrm{~mL})$ and washed with water $(3 \times 50 \mathrm{~mL})$ additional chloroform $(50 \mathrm{~mL})$. The aqueous phase was extracted with additional chloroform $(50 \mathrm{~mL})$ and the combined organic extracts were dried over sodium sulphate. Evaporation of the solvents left $1,62 \mathrm{~g}$ of solid. 


\section{Preparation of TMCTS-DVB-CD(9O-TMS)}

Four solids having a fixed TMCTS/DVB molar ratio of $1 / 3$ was prepared modifying the amount of $\mathrm{CD}$ in $\mathrm{CD} / \mathrm{TMCTS}$ molar ratio of $0,1 / 100,1 / 50$ and $1 / 10$. CD y DVB were mixed at $0{ }^{\circ} \mathrm{C}$ and once the solution reach a constant temperature of $0{ }^{\circ} \mathrm{C}$, TMCTS was injected under continuous stirring, and then the temperature is raised up to $85^{\circ} \mathrm{C}$ and kept at this temperature for $24 \mathrm{~h}$. White solids were obtained and the obtained yield depends on the amount of $\mathrm{CD}$ used in the preparation. The solids obtained were labelled as TMCTSDVB-CD (x) being $\mathrm{x}$ the CD/TMCTS molar ratio, that is, $0 ; 0,01 ; 0,02$ and 0.1 .

\section{Unprotection of $\mathrm{OH}$ group in the hybrids}

The hybrids obtained in the previous step were refluxed in methanol (70 $\mathrm{mL}$ ) for $24 \mathrm{~h}$. The crude product was washed with pentane $(50 \mathrm{~mL})$ filtered and vacuum drying to room temperature.

\section{Preparation of colloidal ruthenium and catalyst}

A total of $5 \mathrm{~mL}$ aqueous solution of $\mathrm{RuCl}_{3} \cdot \mathrm{nH}_{2} \mathrm{O}(0.5 \mathrm{~g})$ was added into to $200 \mathrm{~mL}$ of glycol. Then $15 \mathrm{~mL}$ aqueous solution of $\mathrm{NaOH}(0.05 \mathrm{M})$ was added under stirring. A transparent dark-brown homogeneous colloidal solution of the $\mathrm{Ru}$ metal nanocluster was obtained. Later on, appropriated aliquots of the dispersion were taken off and used to impregnate the hybrid supports in a rotary evaporator to prepare catalysts containing $3 \mathrm{wt} \%$ of Ru. Finally, the obtained solids were washed with $200 \mathrm{~mL}$ of a $0.30 \mathrm{M}$ solution of $\mathrm{NaNO}_{3}$ and then dried under vacuum at room temperature.

\section{Catalysts characterization}

FTIR studies were performed in a Nicolet 6700 spectrophotometer, specific area was evaluated from the $\mathrm{N}_{2}$ adsorption at $77 \mathrm{~K}$ in an automatic Micromeritics system Model ASAP 2010. X-ray diffraction (XRD) patterns were obtained on a Rigaku diffractometer using a $\mathrm{Ni}$ filter and $\mathrm{Cu} \mathrm{K}$ radiation. TEM studies were carried out by using a Jeol Model JEM-1200 EXII System. Photoelectron spectra (XPS) were recorded using an Escalab $200 \mathrm{R}$ spectrometer provided with a hemispherical analyzer, and using non-monochromatic $\mathrm{Mg} \mathrm{K}_{\alpha} \mathrm{X}$-ray radiation $(h \mathrm{v}=1253.6 \mathrm{eV})$. The surface $\mathrm{Ru} / \mathrm{Si}$ and $\mathrm{N} / \mathrm{Si}$ atomic ratios were estimated from the integrated intensities of Ru $3 p, S i 2 p, C$ s and N 1s lines after background subtraction and corrected by the atomic sensitivity factors [14]. The spectra were fitted to a combination of Gaussian-Lorentzian lines of variable proportion. The binding energy of the $\mathrm{Si} 2 \mathrm{p}$ peak at $103.4 \mathrm{eV}$ was taken as an internal standard.

\section{Catalytic activity}

The hydrogenation in liquid phase of ethyl pyruvate was carried out in a stainless steel batch reactor at $25{ }^{\circ} \mathrm{C}$ and a hydrogen pressure of $40 \mathrm{bar}$. The hydrogen pressure was kept constant during the reaction. The stirring speed was keeping constant at $900 \mathrm{rpm}$. The solvent used in this study was cyclohexane (Merck). In all the standard experiments, the volume of solvent in the pressurized batch reactor was $50 \mathrm{~mL}$. Samples were taken periodically from the reactor and analysed by using a gas chromatograph-mass spectrometer (GCMS-QP5050 Shimadzu) provided with a chiral $\beta$-dex 225 column (30 m; Supelco).

\section{RESULTS AND DISCUSSION}

Figure 2 shows the FTIR spectra of TMCTS, CD and the hybrid precursors. It can be seen distinctive adsorption bands for each reactant. Thus, in the region of $1000 \mathrm{~cm}^{-1}$ a strong band corresponding to the $\mathrm{O}-\mathrm{Si}-\mathrm{O}$ bending mode of the TMCTS can be observed. This band decreases strongly as the amount of CD bonded to the precursor increases. Additionally, as the amount of $\mathrm{CD}$ anchored to the support increases the characteristic stretching bands close to $3000 \mathrm{~cm}^{-1}$ also increase, due to they correspond to the $\mathrm{C}-\mathrm{H}$ stretching vibration of the methylene and methyl groups of CD and DVB. It is also relevant to confirm the presence of a band close to $3400 \mathrm{~cm}^{-1}$ attributed to $\mathrm{O}-\mathrm{H}$, which only may appear in these samples by the presence of $\mathrm{CD}$, and therefore this band increases with the $\mathrm{CD}$ content.

Taking into account that the presence of the $\mathrm{OH}$ group of the $\mathrm{CD}$ is absolutely necessary in order to induce the formation of $\mathrm{H}$ bridge with the substrate to favour the induction phenomena, as pointed out Baiker [15], and that during hydrosilylation reactions the attack to the $\mathrm{OH}$ group is strongly favoured, the protection of the $\mathrm{OH}$ group of the $\mathrm{CD}$ was carried as described previously. Moreover, it should be mentioned that the thermodynamic predicts that during the hydrosilylation reactions, the formation of Si-O bonds is favoured and consequently the reaction must be carried out under inert atmosphere [8]. After the TMCTS-DVB-CD samples were prepared, an unprotection treatment, allowed to obtain the hybrid having polymeric macrolattice structures (Fig.3) which contains the chiral inducer anchored by the vinyl group of the cinchonidine, thus recovering the $\mathrm{OH}$ group bonded to de $\mathrm{CD}$ anchored to the lattice.

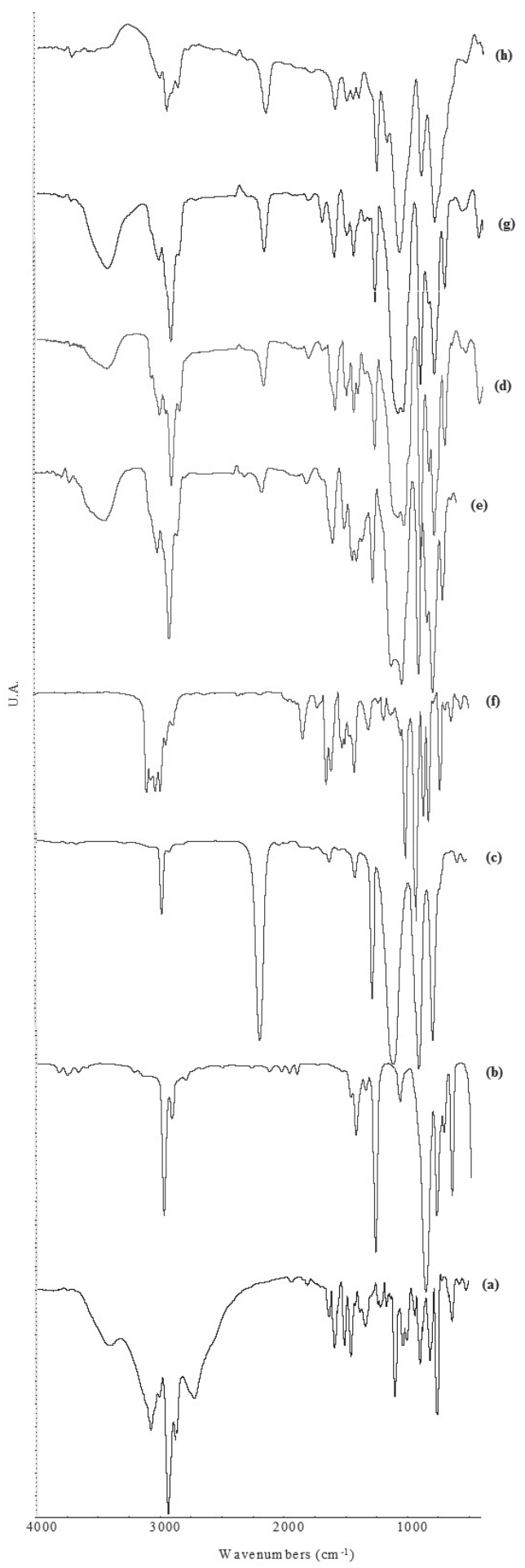

Figure 2. FTIR spectra of: (a) cinchonidine (b) chlorotrimethylsilane (c), 1,3,5,7-tetramethylcycletetrasiloxane (d), divinylbenzene (e) Ru/TMCTSDVB-CD (0.10) (f), Ru/TMCTS-DVB-CD (0.02) (g), Ru/TMCTS-DVB-CD (0.01) (h), Ru/TMCTS-DVB-CD (0.00). 


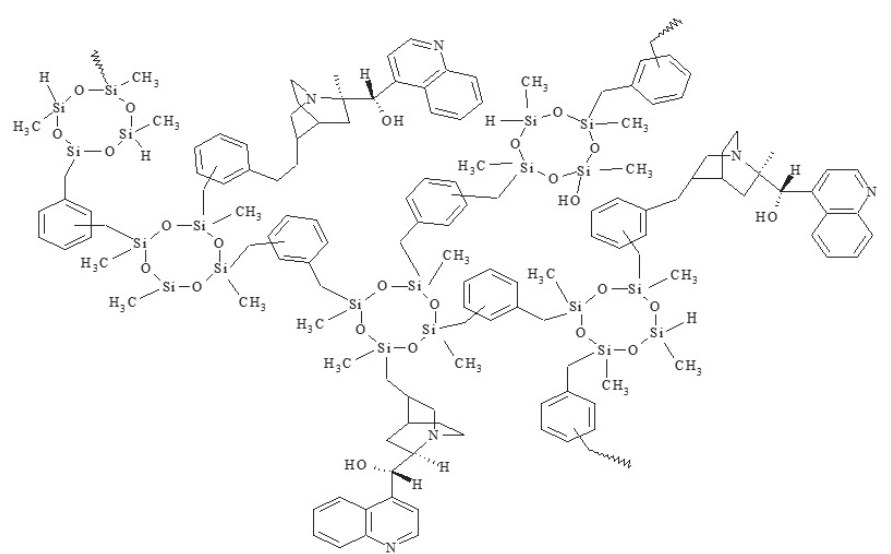

Figure 3. Molecular structure of the hybrids after unprotection.

XRD studies revealed that the support is essentially amorphous. Only lines with weak intensity close to $2 \theta \approx 43^{\circ}$ attributed to Ru (0) can be observed (Fig. 4). The Debye-Scherrer method allowed to estimate the Ru particle size by the broadening of the peak [16], being approximately $13.5 \mathrm{~nm}$ for Ru supported on TMCTS-DVB-CD ( $x=0.00$ and 0.01$)$ and close to $8.5 \mathrm{~nm}$ for those supported on TMCTS-DVB-CD $(x=0.02$ and 0.10 , respectively.

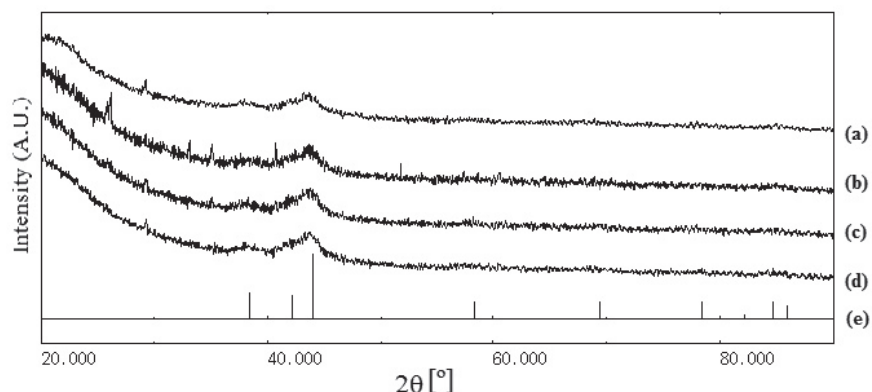

Figure 4. XRD patterns of the $3 \mathrm{wt} . \% \mathrm{Ru}$ supported on the different hybrids: (a) Ru/TMCTS-DVB-CD (0.00); (b) Ru/TMCTS-DVB-CD (0.01); (c) Ru/TMCTS-DVB-CD (0.02); (d) Ru/TMCTS-DVB-CD (0.10); (e) Ru (0) diffraction pattern file $06-0663$.

Metal particle size evaluated by TEM showed only slight differences in the average value, close to $3.7 \mathrm{~nm}$. The particle size distribution in all catalysts is rather narrow with metal particles in the range 1.2 to $10 \mathrm{~nm}$. The significant differences in the particle size values obtained by XRD and TEM are explained considering that the former techniques only gives information of the larger metal particles whereas those smaller than $4 \mathrm{~nm}$ can not be detected and by TEM even high dispersed particle can be observed.

Apparent BET specific area and porosity were evaluated from the nitrogen adsorption isotherms at $77 \mathrm{~K}$ and the results are compiled in Table 1. The $\mathrm{Ru} / \mathrm{TMCTS}-\mathrm{DVB}-\mathrm{CD}(0.00)$ catalyst shows a type II isotherm in the BDDT classification, and no hysteresis loop was observed, indicative of a non porous solid. The other catalysts containing $\mathrm{CD}$ in their structures displayed type IV isotherms with $\mathrm{H} 3$ hysteresis loops, corresponding to parallel sheets type mesopores. The Ru/TMCTS-DVB-CD (0.01) catalyst possesses higher surface area and displays a broader pore radius distribution, centred at $2.2 \mathrm{~nm}$ with significant contribution of pores in the range 2.0 to $20 \mathrm{~nm}$. It is assumed that the insertion of small amount of CD into the TMCTS-DVB structure acts as a pillar in the sheets of the structure material, increasing significantly the surface area and the mesopores which posses the characteristic of slit type pores ( $\mathrm{H} 3$ hysteresis loops). Further increases in the amount of $\mathrm{CD}$ produces a collapse in the pillared type structure and therefore the amount of mesopores and the specific surface area decreases. The catalysts with higher CD content showed a narrower pore radius distribution -in the range 2.0 to $4.0 \mathrm{~nm}$ - indicative of an ordered structure and lower specific area. These adsoption-desorption isotherms data were analysed using the BJH method [17] and the results are displayed in Figure 5 in which the values of $\Delta \mathrm{V} / \Delta \mathrm{r} v s \cdot \log \mathrm{r}_{\mathrm{p}}$ has been plotted.
Table 1. Surface area, metal particle size obtained from XRD and TEM and $\mathrm{Ru} / \mathrm{Si}$ surface atomic ratios obtained from XPS studies of Ru supported catalysts

\begin{tabular}{|l|c|c|c|c|}
\hline \multirow{2}{*}{ Catalyst } & \multirow{2}{*}{$\begin{array}{c}\mathrm{S}_{\text {BET }} \mathrm{N}_{2} \\
\left(\mathrm{~m}^{2} / \mathrm{g}\right)\end{array}$} & \multicolumn{2}{|c|}{$\mathrm{d}, \mathrm{nm}$} & \multirow{2}{*}{$(\mathrm{Ru} / \mathrm{Si})_{\mathrm{s}}$} \\
\cline { 3 - 4 } & 8 & 3.9 & 13.0 & 0.023 \\
\hline $\mathrm{Ru} /$ TMCTS-DVB-CD $(0.00)$ & 8 & 3.7 & 12.6 & 0.014 \\
\hline $\mathrm{Ru} /$ TMCTS-DVB-CD $(0.01)$ & 184 & 3.6 & 8.6 & 0.031 \\
\hline $\mathrm{Ru} /$ TMCTS-DVB-CD $(0.02)$ & 28 & 3.6 & 8.6 & 0.030 \\
\hline
\end{tabular}

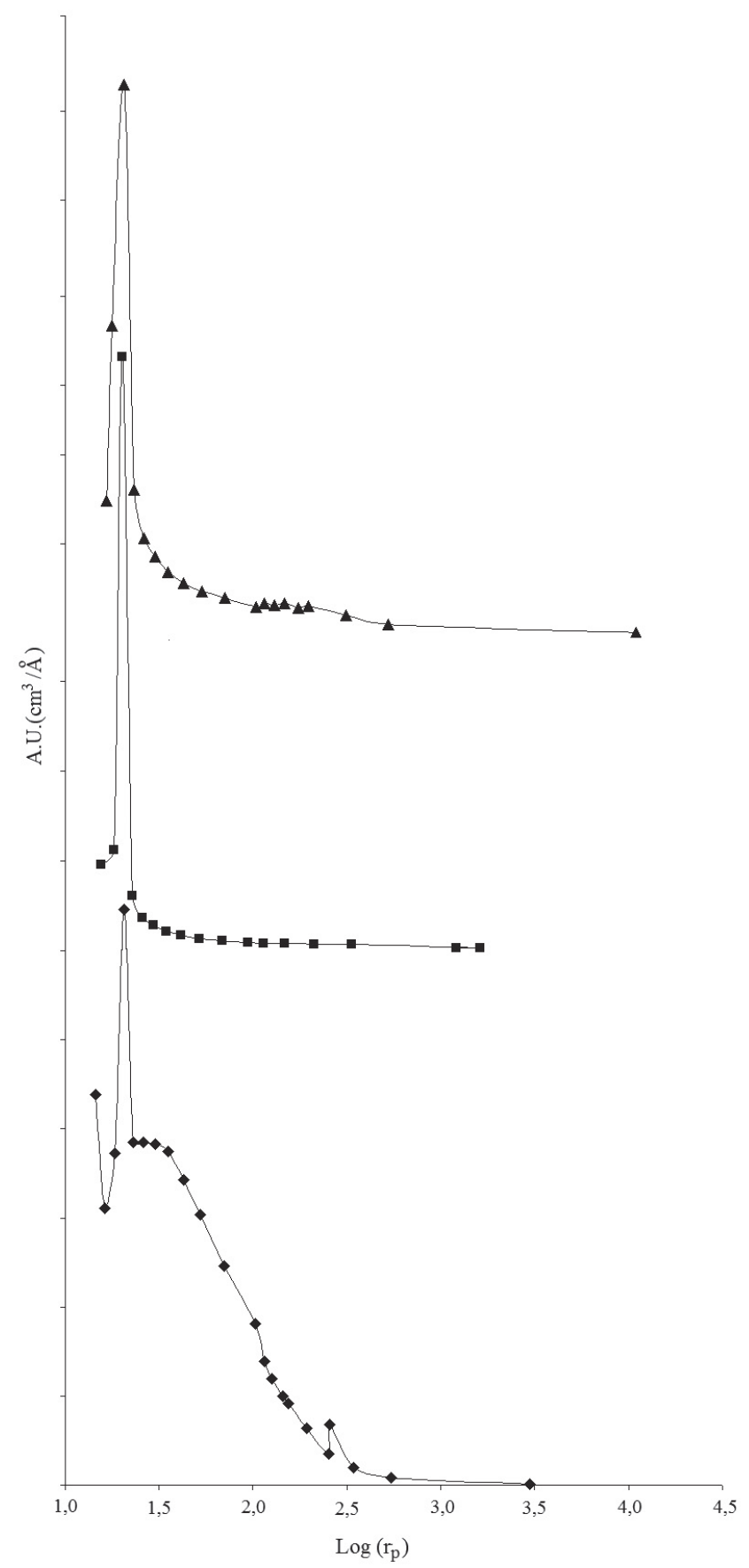

Figure 5. Pore size distribution of Ru/TMTCSDVB-CD catalysts: $\quad$ Ru/TMCTS-DVB-CD (0.01); - Ru/TMCTS-DVB-CD (0.02); $\Delta$ Ru/TMCTS-DVB-CD (0.10). 
XPS studies indicate that Ru is present both, in the metallic state and slightly oxidised species $\left(\mathrm{Ru}^{\delta+}\right.$, see Figure 6) indicative that $\mathrm{Ru}(\mathrm{III})$ was not completely reduced during the formation of the colloid, being the reduced species close to $60 \%$. N 1s core-level showed the presence of two types of nitrogen as expected from the CD structure (BE of 398.8 and $400.8 \mathrm{eV}$, respectively. Similarly, the analyses of C1s core-level spectra revealed two types of C-atoms (BE of 284.8 and $286.6 \mathrm{eV}$ ). With regard to the surface $\mathrm{Ru} / \mathrm{Si}$ atomic ratios (see Table 1), the values are in the range 0.014 to 0.030 comparable with those calculated for the bulk ratios, which are in the range 0.033-0.035. The differences appear in the catalysts with chiral inducer and the one with lower CD concentration and may be explained considering that in the preparation of these hybrids supports the yield is slightly different. As was previously discussed, metal particle size and consequently metal dispersion of all catalysts was similar, therefore no significant differences in the surface $\mathrm{Ru} / \mathrm{Si}$ ratios should be expected.

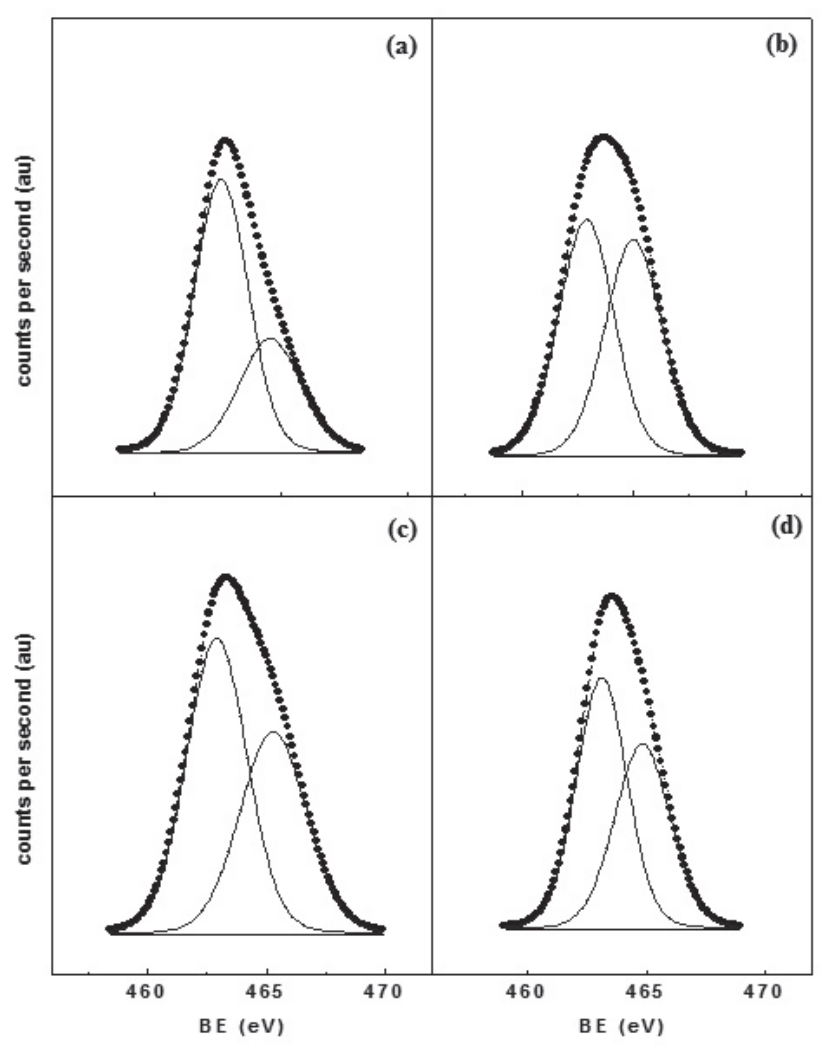

Figure 6. XPS core level spectra of Ru 3p of the Ru supported catalysts: (a)Ru/TMCTS-DVB-CD (0.00); (b)Ru/TMCTS-DVB-CD (0.01); (c) Ru/ TMCTS-DVB-CD (0.02); (d)Ru/TMCTS-DVB-CD $(0.10)$

Catalytic hydrogenation of ethyl pyruvate over the prepared solids is displayed in figure 7 in which the evolution of the conversion level with the reaction time at $298 \mathrm{~K}$ and $40 \mathrm{bar}$, has been plotted. Pseudo first order curves for the four samples is observed, being the catalyst Ru/TMCTS-DVB-CD $(0.00)$, which does not contain $\mathrm{CD}$ in its structure the one that displays the highest activity (conversion level close to $35 \%$ at $2 \mathrm{~h}$ of reaction. Lower conversion levels were shown by the chiral supports which at the same time display conversion levels of $7 ; 5.5$ and 4 mole $\%$ for Ru/TMCTS-DVB-CD (x) catalysts being $\mathrm{x}$ (the $\mathrm{CD} / \mathrm{TMCTS}$ molar ratio) of $0.01,0.02$ and 0.1 , respectively. Two points regarding the activity results should be emphasised: i) The low activity displayed by all the studied catalysts as expected, is attributed to the low hydrogenation ability of ruthenium. ii). Considering that the catalysts posses similar metal particle size, the activity should be essentially the same unless, the porosity of the samples may play an important role. Thus, the differences observed in activity may be explained in terms of the porosity of the support. The ruthenium catalyst supported on the non chiral material (Ru/TMCTSDVB-CD $(0.00))$ is a not porous solid, therefore, the metallic particles remain in the exterior surface of the support. Conversely, in the chiral supports, the metallic particles are distributed in the pore structure of these materials and in those with higher $\mathrm{CD}$ content, a higher fraction of metal crystals remains inside narrow pores limiting the access to the reactant molecules and consequently, reducing the catalytic activity, as can be seen in Figure 7.

The enantiomeric excess (EE) is defined as:

$$
\mathrm{ee}=\frac{[\mathrm{R}]-[\mathrm{S}]}{[\mathrm{R}]+[\mathrm{S}]} \cdot 100 \%
$$

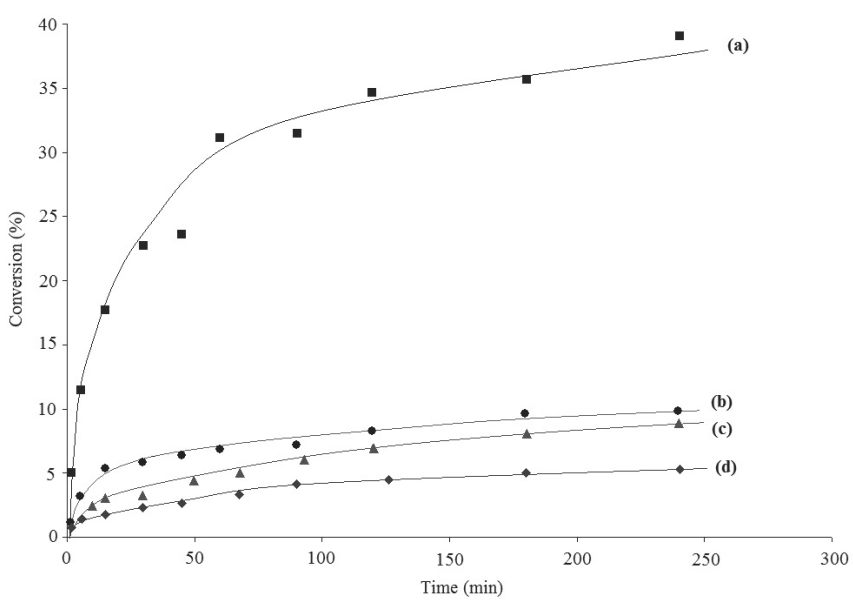

Figure 7. Hydrogenation of ethyl pyruvate over Ru catalysts at 40 bar and $298 \mathrm{~K}$. Evolution of the conversion over time.: (a) Ru/TMCTS-DVB-CD (0.00); (b) Ru/TMCTS-DVB-CD (0.01); (c), Ru/TMCTS-DVB-CD (0.02); (d) $\mathrm{Ru} / \mathrm{TMCTS}-\mathrm{DVB}-\mathrm{CD}(0.10)$.

Figure 8 shows the evolution of the ee with the conversion level of the studied $\mathrm{Ru}$ catalysts. With regard to the enantiomeric excess, the obtained values were rather modest. As expected, no enantiomeric excess was displayed by the achiral catalyst (Ru/TMCTS-DVB-CD $(0.00)$ ). The observed trends indicate that the ee increases with the $\mathrm{CD}$ concentration up to a maximum and then it decreases. Similar trends have been observed for systems using modified noble metal supported catalysts in which the chiral inducer is added to the reaction medium with the substrate. It has been interpreted as follows: at low $\mathrm{CD}$ concentration only the substrate molecules adsorbed in metal-CD interface can induce the formation of the R-enantiomer, being the remaining substrate converted into the racemic mixture, therefore a low ee is obtained. In catalytic systems in which different amount of $\mathrm{CD}$ have been added to the reaction medium to induce chirallity, it has been found that as the $\mathrm{CD}$ concentration increases, the ee is enhanced and after an optimum value it decreases because of $C D$ molecules placed close form a dimer structure which can not induce enantioselectivity. [18]. Thus, each system should display an optimum CD concentration value. It is well known that the metal particle size has an important effect on the ee, however, in the present study the prepared $\mathrm{Ru}$ catalysts have practically the same Ru particle and therefore particle size effect can be ruled out. Additional enhancement of both, the activity and the selectivity can be observed by modification of different preparation procedure and reaction conditions. Thus, using a chiral molecule as stabilizer of the colloid instead glycol or the addition of $\mathrm{CD}$ to the reaction medium allows an important increase in the activity and selectivity levels. Additionally, the $\mathrm{CD}$ quantity inserted in the support lattice requires further improvement [19]. 


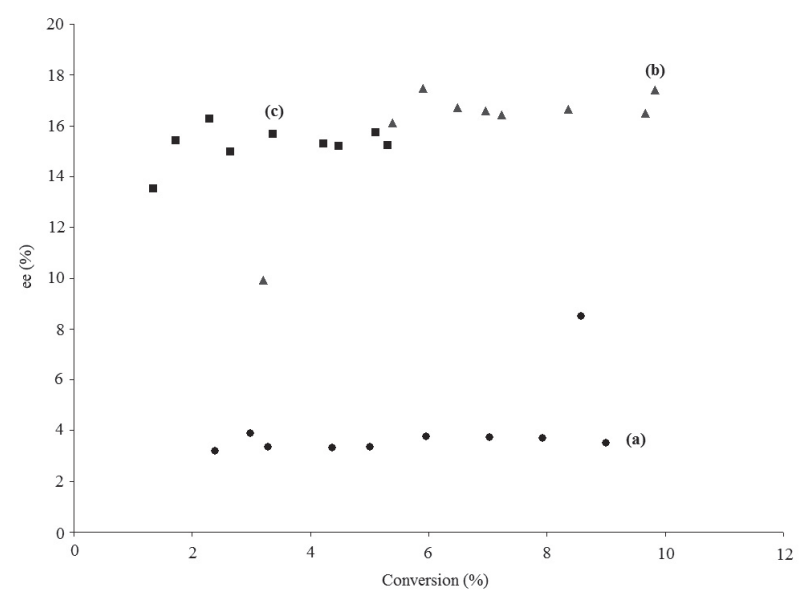

Figure 8. Hydrogenation of ethyl pyruvate over $\mathrm{Ru}$ catalysts at 40 bar and $298 \mathrm{~K}$. Evolution of the ee with the conversion level. $\bullet$ (a) Ru/TMCTSDVB-CD (0.01)); $\boldsymbol{\Delta}$ (b) Ru/TMCTS-DVB-CD and - (c)Ru/TMCTS-DVB$\mathrm{CD}(0.10)$

\section{CONCLUSIONS}

The results indicate that is possible to prepare chiral hybrid supports from the simultaneous silylation of TMCTS with DVB and CD. After impregnation of metal colloid dispersion, chiral catalysts can be obtained. Nitrogen adsorption isotherms revealed that the surface area of the solids depends on the $C D$ concentration inserted into the support lattice. TEM results revealed that the metal particle size distribution is rather narrow, centred close to 4 $\mathrm{nm}$ with almost no differences in the various studied supports. The catalytic results showed that it was possible to induce an enantiomeric excess of the R-lactate, however, further improvements are necessary to obtain competitive enantiomeric catalysts.

\section{ACKNOWLEDGMENT}

The authors thank to CONICYT, FONDECYT Grant 1061001.

\section{REFERENCES}

[1] H. U. Blaser, B. Pugin, and M. Studer, in Chiral catalyst immobilization and recycling, Wiley-VCH, D. E. De Vos, I. F. J. Vankelecom, P. A. Jacobs Eds, Toronto, 1998, 1.

[2] I.F.J. Vankelecom and P.A. Jacobs, in Chiral Catalyst Immobilization and Recycling, Wiley-VCH, Ed. D.E. De Vos, I. F. J. Vankelecom, P. A. Jacobs, Toronto, 1998, 19

[3] E. Song, S.-G. Lee, Chem. Rev., 102, 3495, (2002).

[4] C. Pettersson and C. GioelI, J. Chromat., 398, 247, (1987).

[5] M. Lämmerhofer, W. Lindner, J. Chromat. A, 741, 33, (1996).

[6] A. Lindholm, P. Mäki-Arvela, E. Toukoniitty, T. A. Pakkanen, J. T. Hirvi, T. Salmi, D. Yu. Murzin, R. Sjöholm, and R, Leino, J. Chem., Perkin Trans., 1, 2605, (2002).

[7] I. Busygin, E. Toukoniity, R. Sillampa, D.Y. Murzin, R. Leino, Eur. J. Org. Chem.,2811(2005).

[8] R.O. Pinho, E. Radovanovic, I.L. Torriani, I.V.P. Yoshida, J. Europ. Polym., 40, 2004, 615.

[9] I. Ojima, in The Chemistry of Organic Silicon Compounds, John Wiley \& Sons Ltda., by S. Patai and Z. Rappoport Eds., Chichester, 1998, 1479.

[10] T. Naota, H. Takaya, S. Murahashi, Chem. Rev., 98, 2599, (1998).

[11] L.K. Kurihara, G.M. Chow and P.E. Schoen, NanoStructured Materials, 5-6, 607, (1995).

[12] Y. Wang, J. Ren, K. Deng, L. Gui, and Y. Tang, Chem. Mater., 12, 1622, (2000).

[13] Y. Orito, S. Imai, S. Niwa, G.-H. Nguyen, J. Synth. Org. Chem. Jpn, 37, $173,(1979)$.

[14] P. Reyes, T. Marzialetti, J.L.G. Fierro, Catal. Today, 107-108, 235 , (2005).

[15] A. Baiker, B. Minder, T Mallat, T. Heinz, J. Catal. 154, 371, (1995).

[16] J.R. Anderson, in "Structure of Metallic Catalysts", Academic Press, New York, 1975.

[17] E. P. Barret, L.G. Joyner, P.P. Halenda, J. Am. Chem. Soc. 71, 373, (1951).

[18] E. Orglmeister, T. Mallat, A. Baiker, J. Catal. 233, 333, (2005).

[19] P. Reyes, C. Campos, J.L.G.Fierro, unpublished results. 